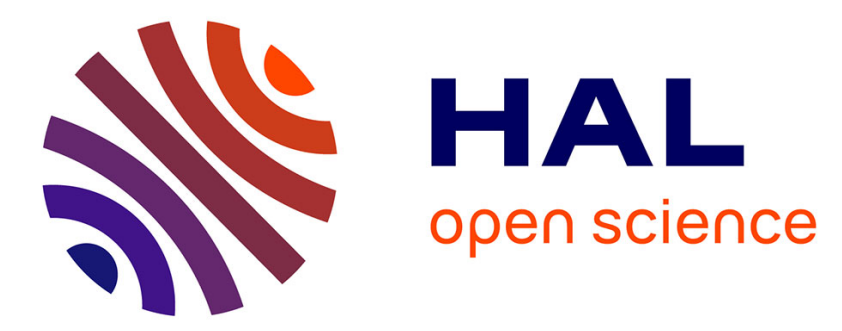

\title{
Practical consensus tracking of multi-agent systems with linear controllers
}

Fayrouz Isfoula, Emmanuel Bernuau, Emmanuel Moulay, Patrick Coirault, Qishuai Liu, Qing Hui

\section{- To cite this version:}

Fayrouz Isfoula, Emmanuel Bernuau, Emmanuel Moulay, Patrick Coirault, Qishuai Liu, et al.. Practical consensus tracking of multi-agent systems with linear controllers. 2019 18th European Control Conference (ECC), Jun 2019, Naples, Italy. pp.3028-3033, 10.23919/ECC.2019.8795734 hal-02266769

\section{HAL Id: hal-02266769 \\ https://hal.science/hal-02266769}

Submitted on 3 Feb 2021

HAL is a multi-disciplinary open access archive for the deposit and dissemination of scientific research documents, whether they are published or not. The documents may come from teaching and research institutions in France or abroad, or from public or private research centers.
L'archive ouverte pluridisciplinaire HAL, est destinée au dépôt et à la diffusion de documents scientifiques de niveau recherche, publiés ou non, émanant des établissements d'enseignement et de recherche français ou étrangers, des laboratoires publics ou privés. 


\title{
Practical consensus tracking of multi-agent systems with linear controllers
}

\author{
Fayrouz Isfoula ${ }^{1}$, Emmanuel Bernuau ${ }^{2}$, Emmanuel Moulay $^{3}$, Patrick Coirault ${ }^{1}$, Qishuai Liu ${ }^{4}$, and Qing Hui ${ }^{4}$
}

\begin{abstract}
The second-order practical consensus tracking of multi-agent systems with a fixed topology and a varying leader's velocity is obtained by using a linear control strategy and the input-to-state stability framework. Then, a new control protocol is proposed where the leader, called a perceptive leader, receives information from its followers. Finally, a study is provided in simulation and on a fleet of mobile robots showing the effectiveness of non perceptive and perceptive strategies in terms of speed of convergence, precision and connectivity maintenance.
\end{abstract}

\section{INTRODUCTION}

Since a decade, the consensus problem of multi-agent systems (MAS) has attracted great attention in many fields, such as biology, physics, robotics, and control engineering [1]-[3]. This is mainly due to its wide applications in distributed formation control. Consensus means the agreement of a group of agents on their common states via local interaction. A variety of topics related to consensus problems have been studied such as consensus with switching topology [4]-[9] or finite-time consensus [10]-[12]. Existing consensus algorithms can be categorized into two classes: consensus without a leader called leaderless consensus or simply consensus and consensus with a leader called consensus tracking where the leader is a special agent whose motion is independent from other agents [1]-[3]. The leader is followed by all of other agents which communicate with each other and receive instructions from the leader. The agents following the leader are called the followers.

Since the seminal work of Jadbabaie [13], several control protocols have been proposed to solve the consensus tracking problem [14]-[22] or the finite time consensus tracking problem [23]-[28] with a given graph topology and constant or varying leader's velocity. In all the previous mention work, the proposed controllers are discontinuous or nonlinear. In our article, it is supposed that we have an undirected

\footnotetext{
${ }^{1}$ Fayrouz Isfoula and Patrick Coirault are with LIAS (EA 6315), Université de Poitiers, 2 rue Pierre Brousse, 86073 Poitiers Cedex 9, France fayrouz.isfoula@univ-poitiers.fr, patrick.coiraulteuniv-poitiers.fr

${ }^{2}$ Emmanuel Bernuau is with GENIAL (UMR INRA 1145), AgroParisTech, 1 Avenue des Olympiades, 91744 Massy Cedex, France emmanuel. bernuaudagroparistech. fr

${ }^{3}$ Emmanuel Moulay is with XLIM (UMR CNRS 7252), Université de Poitiers, 11 bd Marie et Pierre Curie, 86073 Poitiers Cedex 9, France emmanuel.moulay@univ-poitiers.fr

${ }^{4}$ Qishuai Liu and Qing Hui are with Department of Electrical and Computer Engineering, University of Nebraska-Lincoln, NE 68588-0511, USA qishuai.liu@huskers.unl.edu, qing.hui@unl.edu
}

connected graph between the followers, a varying leader's velocity and continuous controllers.

All studies done until now consider a non-perceptive leader being focused only on its motion and whose trajectory is independent of the states of the followers [16], [17], [20], [23], [26], [29], [30]. Firstly, we use in this article a nonperceptive leader which can be real or virtual and which is supposed to track a given preassigned trajectory. Its control input is bounded to obtain the practical consensus tracking of a second-order MAS controlled by a linear controller adapted from the classical linear controller proposed in [1] for the consensus. The proof is based on input-to-state stability notions. The practical consensus means that the states' convergence is not exact but in a ball and it has been studied for instance in [31], [32]. Then, in the second part, we use a perceptive leader that tracks a given preassigned trajectory and receives information from its followers and changes its motion according to the evolution of the fleet. Hence, the control input of the perceptive leader contains two components: the first one is its own control objective ensuring the tracking of a given preassigned trajectory and the second one is composed by the pinned nodes' states. A compromise between the control objective of the perceptive leader and the connectivity maintenance with the followers must be found. A study of the speed of convergence, precision and connectivity maintenance of both strategies with a non perceptive and a perceptive leader is provided in simulation and on a fleet of mobile robots.

The article is organized as follows. After some notations and definitions provided in Section III, the problem statement is given in Section IIII Then, the main results of the paper are developed in Section IV] for a non perceptive and a perceptive leader. Simulation results are presented in Section V An experimental platform of a fleet of four mobile robots is used in Section VI to show the effectiveness of the proposed methods. Finally, a conclusion is reached in Section VII.

\section{NOTATIONS AND DEFINITIONS}

Let us introduce the following notations:

- $\mathbb{R}_{+}=\{x \in \mathbb{R}: x \geq 0\}$, where $\mathbb{R}$ is the set of real numbers.

- We denote $1_{n}=(1, \ldots, 1)^{T} \in \mathbb{R}^{n}$.

- $I_{n}$ denotes the unit matrix of size $n$.

- $|\cdot|$ denotes the absolute value in $\mathbb{R}$ and $\|\cdot\|$ the Euclidean norm on $\mathbb{R}^{n}$. 
- If $A$ is an $m \times n$ matrix and $B$ a $p \times q$ matrix, then the Kronecker product $A \otimes B$ is the $m p \times n q$ block matrix

$$
A \otimes B=\left(\begin{array}{ccc}
a_{11} B & \cdots & a_{1 n} B \\
\vdots & \ddots & \vdots \\
a_{m 1} B & \cdots & a_{m n} B
\end{array}\right) .
$$

- If $P$ is an $n \times n$ real symmetric matrix then:

$-\lambda_{\min }(P)$ is the smallest eigenvalue of the matrix $P$

- $\lambda_{\max }(P)$ is the greatest eigenvalue of the matrix $P$.

- A continuous function $\alpha: \mathbb{R}_{+} \rightarrow \mathbb{R}_{+}$belongs to the class $\mathcal{K}$ if $\alpha(0)=0$ and the function is strictly increasing. A function $\alpha: \mathbb{R}_{+} \rightarrow \mathbb{R}_{+}$belongs to the class $\mathcal{K}_{\infty}$ if $\alpha \in \mathcal{K}$ and it is unbounded.

- A continuous function $\beta: \mathbb{R}_{+} \times \mathbb{R}_{+} \rightarrow \mathbb{R}_{+}$belongs to the class $\mathcal{K} \mathcal{L}$ if $\beta(\cdot, t) \in \mathcal{K}_{\infty}$ for each fixed $t \in \mathbb{R}_{+}$ and if for each fixed $s \in \mathbb{R}_{+}$the function $t \mapsto \beta(s, t)$ is decreasing to 0 .

\section{A. Graph theory}

Let us recall some basic definitions about graph theory given for instance in [1, Appendix B].

Definition 1: An undirected graph $\mathcal{G}=(\mathcal{V}, \mathcal{E})$ consists of a set of vertices $\mathcal{V}=\{1, \ldots, N\}$ and a set of edges $\mathcal{E} \subset \mathcal{V} \times \mathcal{V}$ such that $(i, j) \in \mathcal{E}$ iff $(j, i) \in \mathcal{E}$. An edge $(i, j) \in \mathcal{E}$ in an undirected graph $\mathcal{G}$ represents that vertices $i$ and $j$ obtain information from each other.

Remark 2: An undirected path is a sequence of edges in an undirected graph of the form $\left(i_{1}, i_{2}\right),\left(i_{2}, i_{3}\right), \ldots$

Remark 3: An undirected graph is connected if there is an undirected path between every pair of distinct vertices.

Definition 4: The adjacency matrix of an undirected graph $(\mathcal{V}, \mathcal{E})$ is defined by $\mathcal{A}=\left[a_{i j}\right] \in \mathbb{R}^{N \times N}$ where $a_{i j}=a_{j i}=1$ if $(i, j) \in \mathcal{E}$ and $a_{i j}=0$ otherwise.

Definition 5: The Laplacian matrix of an undirected graph associated with adjacency matrix $\mathcal{A}$ is given as $\mathcal{L}=\left[\ell_{i j}\right] \in$ $\mathbb{R}^{N \times N}$ where $\ell_{i i}=\sum_{j=1, j \neq i}^{N} a_{i j}$ and $\ell_{i j}=-a_{i j}$ if $i \neq j$.

It is clear that the matrices $\mathcal{L}$ and $\mathcal{A}$ are symmetric.

Lemma 6 ([33]): All of the nonzero eigenvalues of the Laplacian matrix $\mathcal{L}$ are real and positive for an undirected graph. Zero is a simple eigenvalue of $\mathcal{L}$ and the associated eigenvector is $1_{N}$ if and only if the undirected graph is connected.

\section{B. Input-to-state stability}

Consider the following system

$$
\dot{x}=f(x, u)
$$

where $f: \mathbb{R}^{n} \times \mathbb{R}^{m} \rightarrow \mathbb{R}^{n}$ is continuously differentiable and satisfies $f(0,0)=0$. Let us denote $\mathcal{L}_{\text {loc }}^{\infty}$ the set of locally essentially bounded functions $u: \mathbb{R} \rightarrow \mathbb{R}^{m}$.

Let us recall the definition of input-to-state stability (ISS) and the definition of ISS Lyapunov function.
Definition 7 ([34]): The system (1) is (globally) input-tostate stable (ISS) if there exist a $\mathcal{K} \mathcal{L}$ function $\beta: \mathbb{R}_{+} \times \mathbb{R}_{+} \rightarrow$ $\mathbb{R}$ and a class $\mathcal{K}$ function $\gamma$ such that, for each input $u \in \mathcal{L}_{\text {loc }}^{\infty}$ and each $x_{0} \in \mathbb{R}^{n}$, the following inequality holds

$$
|x(t)| \leq \beta\left(\left\|x_{0}\right\|, t\right)+\gamma(\underset{s \in[0, t]}{\operatorname{ess} \sup }\|u(s)\|), \quad \forall t \geq 0 .
$$

\section{Problem Statement}

Consider a MAS of $N$ agents labeled $1,2, \ldots, N$ that communicate with each other. We consider a graph $\mathcal{G}$ where the vertices are the agents and two agents are linked by an edge when the communication between them is possible. Through the whole of the paper, the communication topology between the agents satisfies the following assumption.

Assumption 8: The graph $\mathcal{G}$ is undirected and connected. The dynamics of each agent is given by

$$
\left\{\begin{array}{l}
\dot{q}_{i}=p_{i} \\
\dot{p}_{i}=u_{i}
\end{array} \quad i=1, \ldots, N\right.
$$

where $q_{i} \in \mathbb{R}^{m}$ is the position, $p_{i} \in \mathbb{R}^{m}$ is the velocity and $u_{i}$ is the control input of the agent $i$. The agents are called followers and track the trajectory of a special agent called the leader labeled by 0 which has its own control objective. In general, only a small fraction of the followers can communicate with the leader.

Definition 9: The matrix $D$ of a graph $(\mathcal{V}, \mathcal{E})$ with a leader denoted 0 is defined by $D=\operatorname{Diag}\left\{a_{10}, \ldots, a_{N 0}\right\}$, where $a_{i 0}=1$ if the agent $i$ communicate with the leader and $a_{i 0}=0$ otherwise. If $a_{i 0}=1$ then the node $i$ is called a pinned node.

We suppose that we have the following assumption

Assumption 10: The matrix $D$ has at least one non-zero entry.

The dynamics of the leader is given by

$$
\left\{\begin{array}{l}
\dot{q}_{0}=p_{0} \\
\dot{p}_{0}=u_{0}
\end{array}\right.
$$

where $q_{0} \in \mathbb{R}^{m}$ is the position of the leader, $p_{0} \in \mathbb{R}^{m}$ is its velocity and $u_{0}$ is the time varying leader's acceleration. The leader tries to track a preassigned trajectory $\left(q_{\mathrm{ref}}, p_{\mathrm{ref}}\right)$ which dynamics is given by $\dot{q}_{\text {ref }}=p_{\text {ref }}, \dot{p}_{\text {ref }}=u_{\text {ref }}$. Let us stress that this reference trajectory is only known by the leader. The state $\left(q_{\text {ref }}, p_{\text {ref }}\right)$ is also defined as the state of a virtual leader.

Let us now define the practical consensus tracking.

Definition 11 (Practical consensus tracking): The MAS (3) is said to solve the practical consensus tracking problem if there exists $\varepsilon \geq 0$ such that for all $i \in\{0, \ldots, N\}$

$$
\begin{aligned}
& \limsup _{t \rightarrow+\infty}\left\|q_{i}(t)-q_{\mathrm{ref}}(t)\right\| \leq \varepsilon, \\
& \limsup _{t \rightarrow+\infty}\left\|p_{i}(t)-p_{\mathrm{ref}}(t)\right\| \leq \varepsilon,
\end{aligned}
$$

Remark 12: If $\varepsilon=0$, we recover the consensus tracking problem.

The aim of this paper is to design controls $u_{i}, i \in$ $\{0, \ldots, N\}$ such that the system (3)-(4) achieves practical consensus tracking. 


\section{MAIN RESULTS}

We consider the following control protocol of the MAS (3) proposed in [1]

$$
u_{i}=-k_{1} \sum_{j=0}^{N} a_{i j}\left(q_{i}-q_{j}\right)-k_{2} \sum_{j=0}^{N} a_{i j}\left(p_{i}-p_{j}\right),
$$

for $i \in\{1, \ldots, N\}$, where $k_{1}>0$ and $k_{2}>0$ are positive constants, $a_{i j}$ are the components of the adjacency matrix $\mathcal{A}$ and $a_{i 0}$ are the diagonal components of the matrix $D$.

In the sequel, we propose two control laws for the leader. The first one is based only on the leader's state and the reference trajectory (and therefore not on the followers' state), which justifies the terminology of non-perceptive leader used below. On the contrary, the second one takes into consideration the followers' state, hence the name of perceptive leader.

\section{A. The non-perceptive leader case}

In this section, the leader's acceleration is given by

$$
u_{0}=u_{\text {ref }}-k_{1}\left(q_{0}-q_{\text {ref }}\right)-k_{2}\left(p_{0}-p_{\text {ref }}\right)
$$

where $k_{1}>0$ and $k_{2}>0$ are the same constants than in (5). Remark that, if $q_{0}(0)=q_{\text {ref }}(0)$ and $p_{0}(0)=p_{\text {ref }}(0)$, and in absence of external disturbances, we have $q_{0}(t)=q_{\text {ref }}(t)$ and $p_{0}(t)=p_{\text {ref }}(t)$ for all $t \geq 0$. We have the following result.

Theorem 13: Consider a MAS with dynamics (3) under the control protocol (5) and the non-perceptive leader (4) with control (6). Suppose that Assumption 8 and Assumption 10 hold. If there exists $u_{\max }>0$ such that $\sup _{t \geq 0}\left|u_{\text {ref }}(t)\right| \leq u_{\max }$, then the MAS solves the practical consensus tracking problem.

Proof: We define the vectors $e_{q}$ and $e_{p} \in \mathbb{R}^{n}, \quad n=m(N+1)$ by $e_{q}=$ $\left(\left(q_{0}-q_{\text {ref }}\right)^{T},\left(q_{1}-q_{\text {ref }}\right)^{T}, \ldots,\left(q_{N}-q_{\text {ref }}\right)^{T}\right)^{T} \quad$ and $e_{p}=\left(\left(p_{0}-p_{\text {ref }}\right)^{T},\left(p_{1}-p_{\text {ref }}\right)^{T}, \ldots,\left(p_{N}-p_{\text {ref }}\right)^{T}\right)^{T}$. We also define the matrix $M \in \mathbb{R}^{n \times n}$ by blocks:

$$
M=\left(\begin{array}{cc}
-I_{m} & 0_{m \times(N m)} \\
a \otimes I_{m} & -S
\end{array}\right),
$$

where $a=\left(a_{10}, \ldots, a_{N 0}\right)^{T}$ and $S=\left(\mathcal{L}_{f}+D\right) \otimes I_{m}$. We obtain

$$
\left\{\begin{array}{l}
\dot{e}_{q}=e_{p} \\
\dot{e}_{p}=k_{1} M e_{q}+k_{2} M e_{p}-b u_{\text {ref }}
\end{array}\right.
$$

with $b=\left(0_{m}^{T}, 1_{m N}^{T}\right)^{T} \in \mathbb{R}^{n}$ and $\mathcal{L}_{f}$ the Laplacian matrix of the graph of the followers. If we denote $e=\left(e_{q}^{T}, e_{p}^{T}\right)^{T} \in$ $\mathbb{R}^{2 n}, B=-\left(0_{n}^{T}, b^{T}\right)^{T}$ and $A=\left[\begin{array}{cc}0_{n} & I_{n} \\ k_{1} M & k_{2} M\end{array}\right]$, then the system (7) is equivalent to

$$
\dot{e}=A e+B u_{\mathrm{ref}} .
$$

Let us show that all the real parts of the eigenvalues of $A$ are strictly negative. Denoting $\chi_{A}$ the characteristic polynomial of the matrix $A$, we find

$$
\chi_{A}(\lambda)=\operatorname{det}\left(\lambda^{2} I_{n}-k_{2} \lambda M-k_{1} M\right)
$$

and then

$\chi_{A}(\lambda)=$

$\operatorname{det}\left(\begin{array}{cc}\lambda^{2} I_{m}+k_{2} \lambda I_{m}+k_{1} I_{m} & 0_{m \times(m N)} \\ -k_{2} \lambda a \otimes I_{m}-k_{1} a \otimes I_{m} & \lambda^{2} I_{m N}+k_{2} \lambda S+k_{1} S\end{array}\right)$.

We know that $I_{m}$ and $S$ commute. Then, expanding this determinant by minors we get

$$
\chi_{A}(\lambda)=\left(\lambda^{2}+k_{2} \lambda+k_{1}\right)^{m} \operatorname{det}\left(\lambda^{2} I_{m N}+\left(k_{1}+k_{2} \lambda\right) S\right) .
$$

We see that the eigenvalues of $A$ are the roots of the polynomial $\lambda^{2}+k_{2} \lambda+k_{1}$, which are of negative real part provided that $k_{1}>0$ and $k_{2}>0$, and the roots of $\pi(\lambda)=$ $\operatorname{det}\left(\lambda^{2} I_{m N}+(1+\lambda) S\right)$. Note that $\pi\left(-k_{1} / k_{2}\right)=1$ thus $-k_{1} / k_{2}$ is not a root of $\pi$. Therefore we can write that

$$
\begin{aligned}
\pi(\lambda) & =\left(k_{1}+k_{2} \lambda\right)^{m N} \operatorname{det}\left(S+\frac{\lambda^{2}}{k_{1}+k_{2} \lambda} I_{n}\right) \\
& =\left(k_{1}+k_{2} \lambda\right)^{m N} \chi_{S}\left(\frac{\lambda^{2}}{k_{1}+k_{2} \lambda}\right) .
\end{aligned}
$$

Under Assumption 8 and from Lemma 6, the matrix $\mathcal{L}_{f}$ is symmetric positive-definite and $D$ is diagonal with positive entries. Hence $S$ is a symmetric, positive-definite matrix and thus the eigenvalues of $S$ are positive. Let $\mu>0$ be an eigenvalue of $S$. By computing the solutions of the equation $\mu=-\frac{\lambda^{2}}{k_{1}+k_{2} \lambda}$, it follows that the real part of $\lambda$ is always negative and we conclude that $A$ is Hurwitz. It follows that there exist $P$ and $Q$, two symmetric, positive-definite matrices, such that $P A+A^{T} P=-Q$. Consider now the candidate Lyapunov function

$$
V(e)=e^{T} P e .
$$

The time derivative of $V$ is given by

$$
\begin{aligned}
\dot{V} & =\dot{e}^{T} P e+e^{T} P \dot{e} \\
& =\left(A e+B u_{\mathrm{ref}}\right)^{T} P e+e^{T} P\left(A e+B u_{\mathrm{ref}}\right) \\
& =e^{T} A^{T} P e+e^{T} P A e+\left(B^{T} P e+e^{T} P B\right) u_{\mathrm{ref}} \\
& =-e^{T} Q e+2 e^{T} P B u_{\mathrm{ref}} .
\end{aligned}
$$

By common manipulations, we get

$$
\dot{V} \leq \sqrt{e^{T} P e}\left(-c \sqrt{e^{T} P e}+2 u_{\max } \sqrt{B^{T} P B}\right),
$$

with $c=\lambda_{\min }\left(P^{-1 / 2} Q P^{-1 / 2}\right)$. Fix $\varepsilon \in(0,1)$. As long as $\sqrt{e^{T} P e} \geq \frac{2 u_{\max } \sqrt{B^{T} P B}}{c(1-\varepsilon)}$, we have $\dot{V} \leq-\varepsilon c V(e)$ and thus $V(e) \leq \exp (-\varepsilon c t) V(e(0))$. Therefore we have either $V(e) \leq \exp (-\varepsilon c t) V(e(0))$ or $V(e) \leq\left(\frac{2 u_{\max } \sqrt{B^{T} P B}}{c(1-\varepsilon)}\right)^{2}$, that is

$V(e) \leq \max \left\{\exp (-\varepsilon c t) V(e(0)) ;\left(\frac{2 u_{\max } \sqrt{B^{T} P B}}{c(1-\varepsilon)}\right)^{2}\right\}$.

Using $\lambda_{\min }(P)\|e\|^{2} \leq V(e) \leq \lambda_{\max }(P)\|e\|^{2}$ we finally get

$$
\|e\| \leq \max \left\{\frac{\sqrt{\lambda_{\max }(P)}}{\sqrt{\lambda_{\min }(P)}} \exp \left(-\frac{\varepsilon c t}{2}\right)\|e(0)\| ; \gamma u_{\max }\right\}
$$


with $\gamma=\frac{2 \sqrt{B^{T} P B}}{c(1-\varepsilon) \sqrt{\lambda_{\min }(P)}}$. Therefore, the system error $[8$ is ISS with respect to the input $u_{\text {ref. }}$. It leads to

$$
\limsup _{t \rightarrow+\infty}\|e(t)\| \leq \gamma u_{\max }
$$

i.e. the MAS solves the practical consensus tracking problem.

\section{B. The perceptive leader case}

Consider the following assumption on the communication topology between the leader and its followers.

Assumption 14: The paths between the leader and its followers are undirected.

Due to Assumption 14, the MAS (3) can be controlled by a perceptive leader with the following control

$$
\begin{aligned}
u_{0}= & -k_{1}\left(q_{0}-q_{\text {ref }}\right)-k_{2}\left(p_{0}-p_{\text {ref }}\right) \\
& -k_{1} \sum_{i=1}^{N} a_{i 0}\left(q_{0}-q_{i}\right)-k_{2} \sum_{i=1}^{N} a_{i 0}\left(p_{0}-p_{i}\right) .
\end{aligned}
$$

The first part of the controller ensures the tracking after the predefined trajectory and the second part ensures the connectivity between the followers and the leader.

Theorem 15: Consider a MAS with dynamics (3) under the control protocol (5) and the perceptive leader (4) with control (10). Suppose that Assumption 8, Assumption 10 and Assumption 14 hold. If there exists $u_{\max }>0$ such that $\sup _{t \geq 0}\left|u_{\text {ref }}(t)\right| \leq u_{\max }$, then the MAS solves the practical consensus tracking problem.

Proof: Consider the graph $(\bar{V}, \overline{\mathcal{E}})$ obtained by adding the leader to the followers' graph. Given Assumption 14, the graph $(\bar{V}, \overline{\mathcal{E}})$ verifies Assumption 8 . We will now add a "virtual leader" to this MAS. This virtual leader is only linked to the real leader. Hence the $D$ matrix associated with this virtual leader has exactly one non-zero entry at the first line, first column; this yields that Assumption 10 holds. Now, assume that the virtual leader follows the dynamics and control rule defined by (4) and (6) but with initial condition $q_{\text {ref }}(0)$ and $p_{\text {ref }}(0)$. As we already pointed out, this means that the trajectory of the virtual leader will stick to the reference trajectory. Therefore, we see that the control law of the leader (10) corresponds the control law of a follower (5) that is linked with the leader. This means that we can apply Theorem 13 and we conclude that the MAS solves the practical consensus tracking problem.

Remark 16: In the proof of Theorem 15, the dynamics of the virtual leader is only known by the leader. Using this virtual leader is just a technique to prove the theorem and does not need additional online simulations. Moreover, the eventual practical consensus can be improved by considering the following more general control law of the leader

$$
\begin{aligned}
u_{0}= & u_{\text {ref }}-\ell_{1}\left(q_{0}-q_{\text {ref }}\right)-\ell_{2}\left(p_{0}-p_{\text {ref }}\right) \\
& -k_{1} \sum_{i=1}^{N} a_{i 0}\left(q_{0}-q_{i}\right)-k_{2} \sum_{i=1}^{N} a_{i 0}\left(p_{0}-p_{i}\right) .
\end{aligned}
$$

The proof of this fact is similar to the proof of Theorem 13. The gains $\ell_{1}$ and $\ell_{2}>0$ allow the leader to change the balance between following the reference trajectory and waiting for the followers.

\section{Simulations}

In this section, we demonstrate in simulations the effectiveness of the proposed linear controllers for the practical consensus of MAS with both perceptive and non-perceptive leaders. We consider a second-order MAS with $N=10$ agents. All agents are moving in the plane $(m=2)$ with the dynamics given by (3). The leader is denoted by 0 and the graph between the agents is undirected. The network topology is given by Fig. 1. It can be noted that the undirected graph $\mathcal{G}$ is connected and the leader is in the neighborhood of agents 1,2 and 6 .

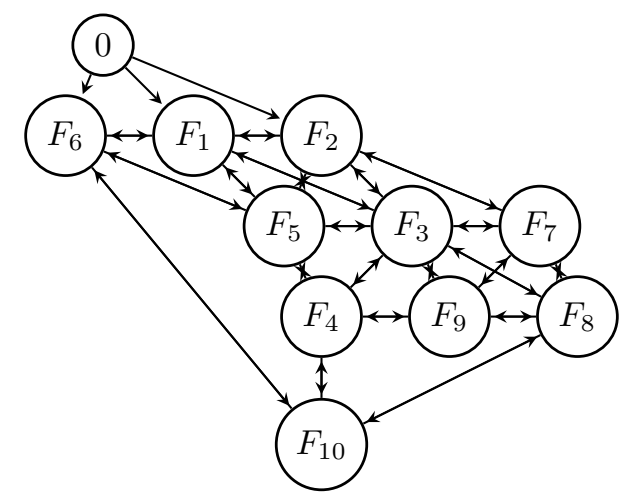

Fig. 1: Topology of the MAS of 10 followers with a leader denoted 0 .

The initial positions of the followers $\left(q_{i}(0)\right.$ are randomly chosen from the box $[-10,10]^{2}$ while the initial velocities are $p_{i}(0)=(0,0)$. The initial position of the leader $q_{0}(0)$ is randomly chosen from the square box $[-10,10]^{2}$ and its initial velocity is $p_{0}(0)=(0,0)$. The simulations are computed with a sampling rate of $0.01 \mathrm{~s}$. We define the barycenter $G$ of the $N$ follower's position $q_{i}(t)$ by $q_{G}(t)=\frac{1}{N} \sum_{i=1}^{N} q_{i}(t)$. Fig. 2 presents the trajectory of the barycenter $G$ and the reference trajectory $q_{\text {ref }}(t)$ of the virtual leader for a non-perceptive leader. The reference trajectory $q_{\text {ref }}(t)$ is chosen as $q_{\text {ref }_{x}}(t)=Q_{x} \cos \left(\omega_{x} t\right)+q_{0, \text { ref }_{x}}$ and $q_{\text {ref }_{y}}(t)=Q_{y} \sin \left(\omega_{y} t\right)+q_{0, \text { ref }_{y}}$ with $\omega_{x}=0.005(2 \pi) \mathrm{rd} / \mathrm{s}$, $\omega_{y}=0.03(2 \pi) \mathrm{rd} / \mathrm{s}, Q_{x}=5$ and $Q_{y}=10$. It can be seen from Fig. 2 and Fig. 3 that the practical consensus is reached, i.e. the followers remain in a closed neighborhood of the virtual leader.

Fig. 4 (resp. Fig. 5) presents the barycenter position $\left(q_{G_{x}}(t), q_{G_{y}}(t)\right)$ (resp. barycenter speed) and the leader position $\left(q_{0_{x}}(t), q_{0_{y}}(t)\right)$ for a perceptive leader with $q_{\text {ref }}=$ $(5,5)$ and $p_{\text {ref }}=(0,0)$. At $t=0$, the perceptive leader and the virtual leader are at the same position. Due to the perceptive part in the control of the leader, the leader leaves its initial position and tends to reach the agents, while these ones tend to reach the leader. Once the leader is in the neighborhood of the agents, the followers and the leader ultimately converge in the vicinity of $q_{\text {ref }}$. 


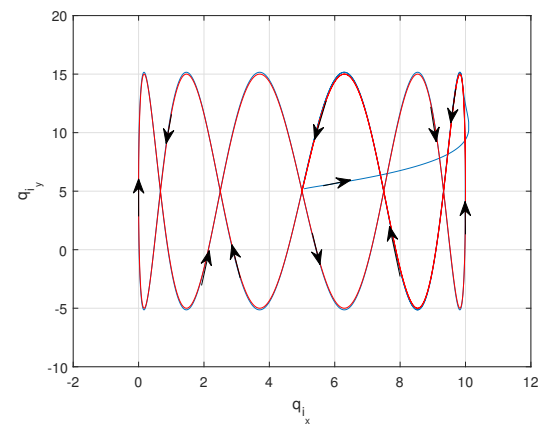

Fig. 2: Barycenter position (blue line) and reference trajectory (red line) for a non-perceptive leader

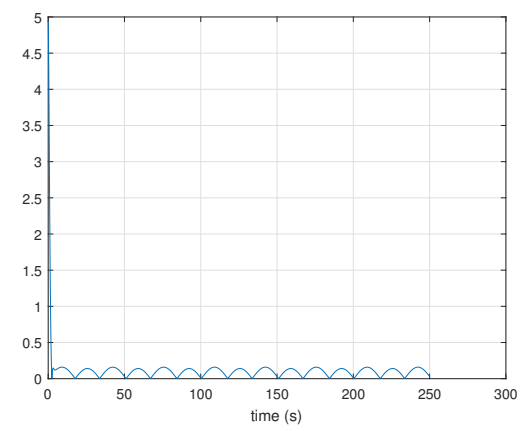

Fig. 3: Position tracking error between the barycenter of the $N$ agents and the position of the virtual leader

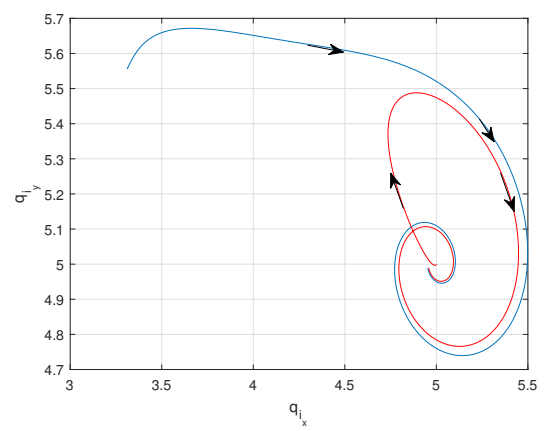

Fig. 4: Barycenter position (blue line) and leader position (red line) for a perceptive leader

\section{EXPERIMENTS}

In this section, we show the effectiveness of the practical consensus of a MAS with both perceptive and non-perceptive leaders via an experimental platform of a fleet of four Pioneer 3-DX mobile robots that use odometer to record their own positions. Their initial positions are given by Fig. 6 The robots can communicate with each other through ethernet with TCP/IP protocols. The control algorithm is written in $\mathrm{C}++$ language and executed at 0.01 s sampling rate. A PC is used to generate the desired trajectory and acts as a virtual leader wheel mobile robot. We approximate the modelling of the robots with a double integrator dynamics (3). Assumption 8 is maintained for this experiment. The

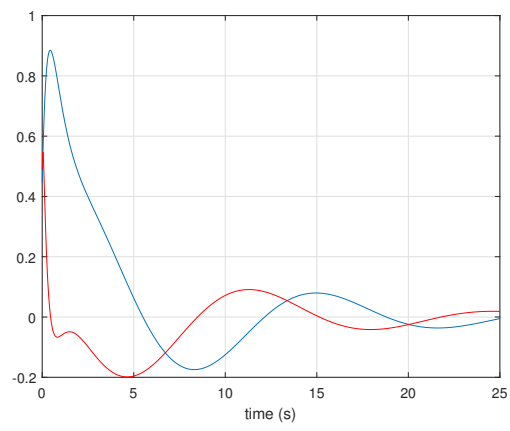

Fig. 5: $x$-barycenter speed (blue line) and $y$-barycenter speed (red line) for a perceptive leader

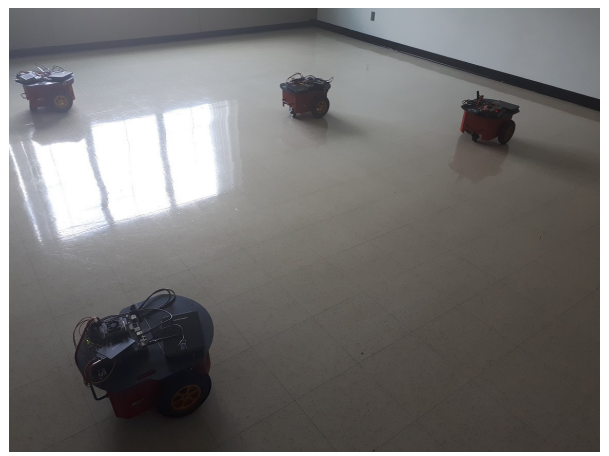

Fig. 6: Initial position of a fleet of four Pioneer 3-DX mobile robots

coordinate of the initial positions of the robots are chosen as $q_{1_{x}}=-0.915 \mathrm{~m}, q_{1_{y}}=0.915 \mathrm{~m} ; q_{2_{x}}=2.205 \mathrm{~m}, q_{2_{y}}=0 \mathrm{~m}$; $q_{3_{x}}=1.22 \mathrm{~m}, q_{3_{y}}=1.22 \mathrm{~m} ; q_{4_{x}}=-0.62 \mathrm{~m}, q_{4_{y}}=2.44 \mathrm{~m}$ and all the velocities are initiated by zero. Moreover, we set $q_{\text {ref }}=p_{\text {ref }}=(0,0)$. In practical applications, robots have limited speed so gains must be chosen small enough so that the speed limit does not exceed. We take $k_{1}, k_{2}, l_{1}, l_{2}=0.3$.

The evolution of the $x$-position of the fleet with controller (5) and the non-perceptive leader (4) is given by Fig. 7

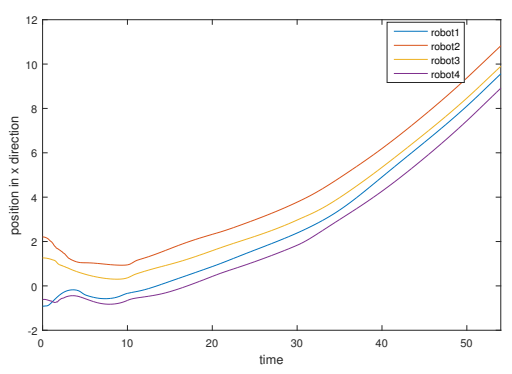

Fig. 7: The evolution of the $x$-position of the fleet with a non-perceptive leader

The evolution of the $x$-position of the fleet with controller (5) and the perceptive leader (10) is given by Fig. 8 .

The practical consensus tracking is said to be achieved when the robots are $1 \mathrm{~m}$ away from the leader and the deviation between the leader and the followers is the angle 


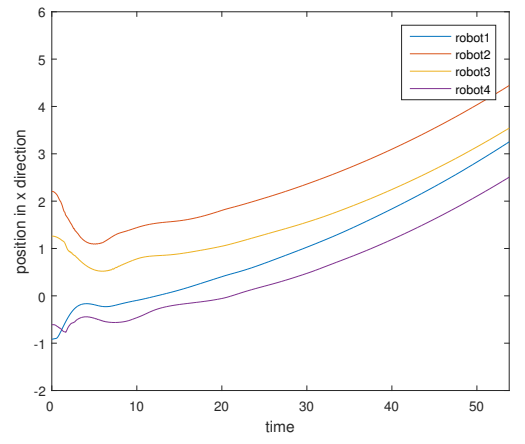

Fig. 8: The evolution of the $x$-position of the fleet with a perceptive leader

of $\frac{\pi}{4}$. As in simulations, we observe that the use of a non perceptive and perceptive leader can ensure the practical consensus with interesting results in terms of speed of convergence, precision and connectivity maintenance.

\section{CONCLUSION}

In this article, the practical consensus tracking of secondorder multi-agent systems under a fixed topology and a varying leader's velocity has been studied. Two cases of practical consensus tracking were investigated, with a non-perceptive leader and with a perceptive leader. It was proved that the practical consensus tracking can be achieved in simulation and on a fleet of mobile robots for both approaches. The case of switching topology has been anticipated as future work.

\section{REFERENCES}

[1] W. Ren, R. W. Beard, Distributed consensus in multi-vehicle cooperative control, Communications and Control Engineering, Springer, 2008.

[2] K.-K. Oh, M.-C. Park, H.-S. Ahn, A survey of multi-agent formation control, Automatica 53 (2015) 424-440.

[3] W. Ren, Y. Cao, Distributed coordination of multi-agent networks: emergent problems, models, and issues, Communications and Control Engineering, Springer, 2011.

[4] L. Cheng, Y. Wang, Z.-G. Hou, M. Tan, Z. Cao, Sampled-data based average consensus of second-order integral multi-agent systems: Switching topologies and communication noises, Automatica 49 (5) (2013) 1458-1464.

[5] Y. Gao, L. Wang, Sampled-data based consensus of continuous-time multi-agent systems with time-varying topology, IEEE Transactions on Automatic Control 56 (5) (2011) 1226-1231.

[6] R. Olfati-Saber, R. M. Murray, Consensus problems in networks of agents with switching topology and time-delays, IEEE Transactions on Automatic Control 49 (9) (2004) 1520-1533.

[7] F. Xiao, L. Wang, Asynchronous consensus in continuous-time multiagent systems with switching topology and time-varying delays, IEEE Transactions on Automatic Control 53 (8) (2008) 1804-1816.

[8] J. Qin, H. Gao, W. X. Zheng, Second-order consensus for multi-agent systems with switching topology and communication delay, Systems \& Control Letters 60 (6) (2011) 390-397.

[9] U. Munz, A. Papachristodoulou, F. Allgower, Consensus in multiagent systems with coupling delays and switching topology, IEEE Transactions on Automatic Control 56 (12) (2011) 2976-2982.

[10] Y. Cao, W. Yu, W. Ren, G. Chen, An overview of recent progress in the study of distributed multi-agent coordination, IEEE Transactions on Industrial Informatics 9 (1) (2013) 427-438.

[11] S. Li, H. Du, X. Lin, Finite-time consensus algorithm for multi-agent systems with double-integrator dynamics, Automatica 47 (8) (2011) 1706-1712.
[12] X. Wang, Y. Hong, Finite-time consensus for multi-agent networks with second-order agent dynamics, IFAC Proceedings Volumes 41 (2) (2008) 15185-15190, 17th IFAC World Congress.

[13] A. Jadbabaie, J. Lin, A. S. Morse, Coordination of groups of mobile autonomous agents using nearest neighbor rules, IEEE Transactions on Automatic Control 48 (6) (2003) 988-1001.

[14] Y. Cao, W. Ren, Distributed coordinated tracking with reduced interaction via a variable structure approach, IEEE Transactions on Automatic Control 57 (1) (2012) 33-48.

[15] J. Fu, J. Wang, Adaptive consensus tracking of high-order nonlinear multi-agent systems with directed communication graphs, International Journal of Control, Automation and Systems 12 (5) (2014) 919-929.

[16] Y. Hong, G. Chen, L. Bushnell, Distributed observers design for leader-following control of multi-agent networks, Automatica 44 (3) (2008) 846-850.

[17] Z. Li, X. Liu, W. Ren, L. Xie, Distributed tracking control for linear multiagent systems with a leader of bounded unknown input, IEEE Transactions on Automatic Control 58 (2) (2013) 518-523.

[18] Z. Li, W. Ren, X. Liu, L. Xie, Distributed consensus of linear multiagent systems with adaptive dynamic protocols, Automatica 49 (7) (2013) 1986-1995.

[19] Z. Meng, W. Ren, Y. Cao, Z. You, Leaderless and leader-following consensus with communication and input delays under a directed network topology, IEEE Transactions on Systems, Man, and Cybernetics, Part B (Cybernetics) 41 (1) (2011) 75-88.

[20] W. Ni, D. Cheng, Leader-following consensus of multi-agent systems under fixed and switching topologies, Systems \& Control Letters 59 (3) (2010) 209-217.

[21] Q. Song, J. Cao, W. Yu, Second-order leader-following consensus of nonlinear multi-agent systems via pinning control, Systems \& Control Letters 59 (9) (2010) 553-562.

[22] Z. Zhang, L. Zhang, F. Hao, L. Wang, Leader-following consensus for linear and lipschitz nonlinear multiagent systems with quantized communication, IEEE Transactions on Cybernetics 47 (8) (2017) 1970-1982.

[23] Z.-H. Guan, F.-L. Sun, Y.-W. Wang, T. Li, Finite-time consensus for leader-following second-order multi-agent networks, IEEE Transactions on Circuits and Systems I: Regular Papers 59 (11) (2012) 26462654.

[24] F. Sun, J. Chen, Z.-H. Guan, L. Ding, T. Li, Leader-following finitetime consensus for multi-agent systems with jointly-reachable leader, Nonlinear Analysis: Real World Applications 13 (5) (2012) 22712284

[25] H. Wang, W. Yu, G. Wen, G. Chen, Fixed-time consensus tracking of multi-agent systems under a directed communication topology, in: 12th IEEE International Conference on Control and Automation, 2016, pp. 186-191.

[26] Y. Zhao, Z. Duan, G. Wen, Y. Zhang, Distributed finite-time tracking control for multi-agent systems: an observer-based approach, Systems \& Control Letters 62 (1) (2013) 22-28.

[27] Y. Zhao, Z. Duan, G. Wen, G. Chen, Distributed finite-time tracking for a multi-agent system under a leader with bounded unknown acceleration, Systems \& Control Letters 81 (2015) 8-13.

[28] Z. Zuo, B. Tian, M. Defoort, Z. Ding, Fixed-time consensus tracking for multi-agent systems with high-order integrator dynamics, IEEE Transactions on Automatic Control 63 (2) (2018) 563-570.

[29] Z. Zuo, B. Tian, M. Defoort, Z. Ding, Fixed-time consensus tracking for multiagent systems with high-order integrator dynamics, IEEE Transactions on Automatic Control 63 (2) (2018) 563-570.

[30] W. Cao, J. Zhang, W. Ren, Leader-follower consensus of linear multiagent systems with unknown external disturbances, Systems \& Control Letters 82 (2015) 64-70.

[31] L. Ding, W. X. Zheng, Network-based practical consensus of heterogeneous nonlinear multiagent systems, IEEE Transactions on Cybernetics 47 (8) (2017) 1841-1851.

[32] X. Dong, J. Xi, Z. Shi, Y. Zhong, Practical consensus for high-order linear time-invariant swarm systems with interaction uncertainties, time-varying delays and external disturbances, International Journal of Systems Science 44 (10) (2013) 1843-1856.

[33] R. Olfati-Saber, J. A. Fax, R. M. Murray, Consensus and cooperation in networked multi-agent systems, Proceedings of the IEEE 95 (1) (2007) 215-233.

[34] E. D. Sontag, Y. Wang, On characterizations of the input-to-state stability property, Systems \& Control Letters 24 (5) (1995) 351-359. 